\title{
REVISITING NICHE FUNDAMENTALS WITH TUKEY DEPTH
}

J. Orestes Cerdeira, Tiago Monteiro-Henriques, M. João Martins, Pedro C. Silva, Diogo Alagador, Aldina M. A. Franco, Manuel L. Campagnolo, Pedro Arsénio, Francisca C. Aguiar, Mar Cabeza

\section{Jorge Orestes Cerdeira}

Departamento de Matemática and Centro de Matemática e Aplicações, Faculdade de

Ciências e Tecnologia, Universidade Nova de Lisboa, Quinta da Torre, 2829-516

Caparica, Portugal.

jo.cerdeira@fct.unl.pt

\section{Tiago Monteiro Henriques}

Centro de Estudos Florestais, CEF, Instituto Superior de Agronomia, Universidade de Lisboa, Tapada da Ajuda, 1349-017 Lisboa, Portugal.

Centro de Investigação e de Tecnologias Agroambientais e Biológicas, CITAB, Universidade de Trás-os-Montes e Alto Douro, Apartado 1013, 5001-801 Vila Real, Portugal.

tmh@isa.ulisboa.pt

\section{João Martins}

Centro de Estudos Florestais, CEF, Instituto Superior de Agronomia, Universidade de Lisboa, Tapada da Ajuda, 1349-017 Lisboa, Portugal.

mjmartins@isa.ulisboa.pt

\section{Pedro C. Silva}

Centro de Estudos Florestais, CEF, Instituto Superior de Agronomia, Universidade de Lisboa, Tapada da Ajuda, 1349-017 Lisboa, Portugal.

pcsilva@isa.ulisboa.pt

\section{Diogo Alagador}

CIBIO/InBio-UE: Centro de Investigação em Biodiversidade e Recursos Genéticos, Universidade de Évora, 7000-890 Évora, Portugal.

alagador@uevora.pt

\section{Aldina M. A. Franco}

School of Environmental Sciences, University of East Anglia, Norwich NR4 7TJ, UK. a.franco@uea.ac.uk

\section{Manuel L. Campagnolo}

Centro de Estudos Florestais, CEF, Instituto Superior de Agronomia, Universidade de Lisboa, Tapada da Ajuda, 1349-017 Lisboa, Portugal.

mlc@isa.ulisboa.pt

\section{Pedro Arsénio}


Linking Landscape, Environment, Agriculture and Food, LEAF, Instituto Superior de Agronomia, Universidade de Lisboa,Tapada da Ajuda, 1349-017 Lisboa, Portugal.

arseniop@isa.ulisboa.pt

\section{Francisca C. Aguiar}

Centro de Estudos Florestais, CEF, Instituto Superior de Agronomia, Universidade de Lisboa, Tapada da Ajuda, 1349-017 Lisboa, Portugal.

fraguiar@isa.ulisboa.pt

\section{Mar Cabeza}

Centre of Excellence in Metapopulation Research, Department of Biosciences, University of Helsinki, Finland.

mar.cabeza@helsinki.fi

Corresponding author: Jorge Orestes Cerdeira

Type of article: Research article

Running headline: Revisiting niche fundamentals 


\section{ABSTRACT}

2 1. The first attempts to describe species ecological niches were simple geometric procedures that depict the niche boundaries directly from environmental data. The convex hull was one of such procedures, popular for its simplicity, clear ecological rational and precise definition of the niche. However, it lacked the ability to differentiate areas of the niche with different probabilities of occurrence according to environmental suitability.

2. We incorporate the Tukey depth, a mathematical tool to measure the centrality of a point within a cloud of points on a multidimensional space, in the convex hull approach to (i) propose a new procedure (CH-Tukey) to estimate species' environmental suitability, and (ii) estimate niche overlap coherently. In addition to a clear ecological rational and simplicity the $\mathrm{CH}$-Tukey procedure has a number of attractive features: use of presence-only data; independence from background data; invariance to scale; robustness to outliers; and the decomposition of the niche into a finite number of iso-suitability levels, permitting the computation of consistent overlap indices. We illustrate the use of CH-Tukey, using occurrence data of the main Quercus species and subspecies from Western Mediterranean Europe, comparing its outputs with BIOCLIM and MaxEnt.

3. Results showed distinct niche geometries among the different approaches. BIOCLIM produced rectilinear niches reflecting the assumption that ecological variables are independent in their action on the species. $\mathrm{CH}-\mathrm{Tukey}$, relaxing this assumption, adjusts niche outer boundary and the inner suitability levels to the 
10 Keywords: Depth functions, environmental envelope, niche modelling, geometric

11 procedures, Hutchinsonian niche, niche overlap, Quercus species.

\section{INTRODUCTION}

14 Hutchinson's niche concept (Hutchinson, 1957) inspired ecological analyses immediately

15 after its proposal (Whittaker \& Levin, 1975), with numerous applications and methods

16 emerging after Hutchinson's seminal paper. Applications are broad, including: studies of

17 resource partitioning in ecological communities (Schoener, 1974), methods on predictive

18 vegetation mapping (Franklin, 1995), explorations of the relation between genome

19 changes and niche differentiation (Rocap et al., 2003), reflections on plant coexistence

20 theories (Silvertown, 2004), or even in taxonomy (Raxworthy et al., 2007). Several other

21 associated ecological concepts followed from Hutchinson's niche concept, e.g.: the guild

22 (Root, 1967), niche breadth and niche overlap (see Colwell \& Futuyma,1971; Hurlbert, 
1 1978), niche width (see Roughgarden, 1972), the regeneration niche (Grubb, 1977), the

2 ontogenetic niche (Werner \& Gilliam, 1984), persistence niche (Bond \& Midgley, 2001),

3 niche conservatism/dynamics (Peterson et al., 1999; Pearman et al., 2008). The uses of

4 the Hutchinson's n-dimensional hypervolume concept across ecology and evolution have

5 been recently surveyed and discussed by Blonder (2017).

6 In recent years, the most conspicuous application of the niche concept has been the

7 plethora of methods developed to estimate the suitability of ecological conditions to

8 species, or eventually, species distributions (Guisan \& Zimmermann, 2000; Franklin,

9 2010; Ahmed et al., 2015). Generalized Linear Models (GLM), Generalized Additive

10 Models (GAM), Maximum Entropy Modelling (MaxEnt), Mahalanobis distance, point-

11 to-point using Gower metric (DOMAIN), Ecological-Niche Factor Analysis (ENFA),

12 among others, produce continuous predictions, raising further challenges. For instance,

13 they depend on thresholds to delineate a precise niche boundary (see Elith et al., 2006;

14 Tsoar et al., 2007; Blonder et al., 2014). Some methods, such as GLM, GAM, MaxEnt,

15 Genetic Algorithm for Rule-set Production (GARP) and artificial neural networks, even

16 after applying user-defined suitability/probability thresholds, may produce unlimited

17 hypervolumes (see Blonder et al., 2014). Although some of those methods fit data well,

18 they may exhibit species-environment relationships with lack of ecological plausibility

19 (Brewer et al., 2016). Addressing this critical issue and aiming at a better relationship

20 with ecological theory, Brewer et al. (2016) proposed a new parametric approach, the

21 Plateau climate envelope. A non-parametric procedure, also ecologically meaningful, is

22 BIOCLIM, the very first geometric approach to the niche. Revisiting and improving such

23 geometric approaches can result interesting, as will be shown hereafter. 
1 BIOCLIM (Nix, 1986; Busby, 1991) was one of the first attempts to formalize the

2 Hutchinsonian niche of a species and to produce maps of species potential distributions in

3 a systematic way (Booth et al., 2014). It is a simple geometric procedure, which identifies

4 the range of suitable values for each environmental variable, independently of the other

5 variables, thus, defining the niche as the hyperrectangle bounded by the minimum and

6 maximum values of the environmental variables for the species known occurrences. This

7 "range" notion within each environmental variable is implicit in Hutchinson's article and

8 is central to the niche concept (i.e. the species is expected to survive and reproduce within

9 some known pair of limiting values for each variable, and not outside those conditions).

10 To distinguish suitability levels within the niche, Nix (1986) and Busby (1991) used the

11 nested hyperrectangles obtained from percentiles of each environmental variable

12 individually. In this way they incorporated Hutchison's perception of an optimal part of

13 the niche versus a suboptimal one near the boundaries (Hutchinson, 1957). The most

14 suitable regions are those corresponding to the environmental conditions lying in the

15 innermost hyperrectangles.

16 The simple approach of BIOCLIM exhibits some attractive properties: i) performs

17 geometric operations with straightforward ecological interpretation; ii) delineates precise

18 (limited) boundaries of the niche; iii) only uses presences and does not depend on the

19 background; iv) is invariant to scale (suitability levels do not change under linear

20 transformations of data); v) is robust to outliers (while the outer regions are sensitive to

21 outliers their influence vanishes for the interior); and vi) produces ordinal predictions

22 decomposing the niche into a finite number of iso-suitability regions. 
1 BIOCLIM presents however a major drawback: it relies on the assumption that ecological

2 variables are independent in their action on the species. With the aim of relaxing this

3 independence assumption, the HABITAT procedure (Walker \& Cocks, 1991) replaced

4 the bounding box envelop used in BIOCLIM by the convex hull of the occurrence points.

5 The convex hull of a set of points defines the minimum convex hypervolume containing

6 all points. Hereafter the construction of the convex hull of occurrences in the

7 environmental space will be referred as the $\mathrm{CH}$-approach. The $\mathrm{CH}$-approach is a

8 comprehensible multidimensional geometric way to circumscribe the niche, but no

9 geometric procedure was used to discriminate suitability levels within it. However, this is

10 achievable in a similar way to the percentile procedure implemented in BIOCLIM by using

11 depth functions, which are procedures to measure the centrality of a point within a data

12 cloud on a multi-dimensional space (Zuo \& Serfling, 2000). Cerdeira et al. (2014)

13 suggested the use of depth functions to estimate suitability levels for species in the $\mathrm{CH}$ -

14 approach. Here we describe and discuss a new method that adds normalized Tukey depth

15 to the $\mathrm{CH}$-approach, and give an application of this proposal to Quercus taxa occurring in

16 the Iberian Peninsula, illustrating it further by assessing the overlap of the produced

17 climatic niches. Finally we compare the geometry of the environmental niches produced

18 by $\mathrm{CH}$-approach using Tukey depth with those of BIOCLIM and MaxEnt.

\section{METHODOLOGIES}

\section{Tukey depth}

22 The Tukey depth of a point (also called location depth or halfspace depth) is a measure,

23 introduced by Tukey (1975), to generalize the order of quantiles for the multivariate case. 
1 Consider a set $P$ of $k$ points of an $n$-dimensional space $(\mathbb{R})$, with $>$, and an arbitrary

2 point $q$. The Tukey depth of point $q$ with respect to $P$ is defined as $d_{q}(P)=m / k$, where $m$ is

3 the minimum number of points to remove from $P$ so that $q$ is not in the convex hull of the

4 remaining points of $P$. We call $m$ the (Tukey) interiority of point $q$ in $P$.

6 Figure 1 depicts a set $P$ of 20 points of $\mathbb{R}$ represented by “ ", and three points $x, y$ and

$7 z$. The lines passing through each of the points $x, y$ or $z$, show that removing 1,3 and 9

8 points from $P$ (those that are on one of the sides of the line thought the point) will leave $x$,

$9 y$ and $z$ outside the convex hull of remaining points of $P$ (those that are on the other side

10 of the line). It can be easily verified that removing less points would leave $x, y$ or $z$,

11 respectively, inside the convex hull of the remaining points of $P$. We can therefore

12 conclude that the interiorities of points $x, y$ and $z$ are 1,3 and 9 , respectively, and $d_{x}(P)=$

$13 \quad 1 / 20, d_{y}(P)=3 / 20$, and $d_{z}(P)=9 / 20$.

14 From the definition, it follows that the maximum interiority cannot exceed

$$
=\frac{-+1}{2}
$$

15 A point $q$ with maximum interiority is a Tukey median point. Point $z$ has interiority 9

16 and, since $M=9$, it is a Tukey median of $P$.

17 Depth functions define a partition of $\mathbb{R}$ into iso-depth regions, i.e., regions of points

18 having equal depth values. Figure $2 \mathrm{~A}$ shows the iso-depth regions corresponding to the

19 set of 10 points of $\mathbb{R}$ represented by “" ". Note that the regions of points with Tukey

20 depth greater than or equal to $m / k$, , are nested, i.e., $\mathbb{R}=\supset \supset \cdots \supset$, 
1 with increasing depth toward the innermost region. The region $\quad(m \geq 1)$ could

2 alternatively be defined as the intersection of all convex hulls of the subsets of $P$ with $k$ -

$3(m-1)$ points.

4 Depth values of the points of a set $P$ can give indication on how $P$ is arranged,

5 distinguishing between configurations of points that are concentrated in the "interior",

6 from those which occur mainly on the "margins". Silva et al. (2014) have recently

7 established that, if the points in $P$ are uniformly distributed, which is a borderline

8 between the two above configurations, the average $d_{q}(P)$, for $q$ in $P$, approximates $1 / 2^{n+1}$.

9 This value, which only depends on the dimension $(n)$ of the representation space,

10 provides a sound threshold to distinguish configurations where the concentration is in the

11 "interior" (average $d_{q}(P)>1 / 2^{n+1}$ ), from configurations where points occur mainly on the

12 "margins" (average $\left.d_{q}(P)<1 / 2^{n+1}\right)$.

\section{Assessing environmental suitability}

15 Let $P$ be the set of $k$ points in $\mathbb{R}$, each point indicating the $n$ environmental conditions of

16 a location where a species occurs. To every point $q$ of $P$ we calculate the $\operatorname{depth} d_{q}(P)$ and

17 divide it by $M / k$. With this rescaling, points with interiority $M$ (if any) score 1 . We

18 propose to interpret these normalized depth values as a measure of environmental

19 suitability for the species. The value 1 corresponds to environmental conditions of sites

20 that belong to the most inner region of the estimated niche. Note that such

21 environmental conditions might not exist. 
1 We call this the CH-Tukey procedure. Under the equilibrium assumption, areas of greater

$2 d_{q}(P)$ values correspond to the optimal parts of the niche (sensu Hutchinson 1957), i.e.

3 areas where species performance is expected to be higher (e.g. growth rate or species

4 fitness). The innermost region, where $d_{q}(P)$ reach its maximum value, is the set of Tukey

5 median points.

6 It should be clear that $\mathrm{CH}$-Tukey procedure assumes unimodal species responses, in

7 relation to the considered environmental variables. More specifically, $\mathrm{CH}-\mathrm{Tukey}$

8 procedure assumes that the suitability of every point of the line connecting two points $q_{1}$

9 and $q_{2}$ of the environmental space, will be at least as large as the minimum between the

10 suitability of $q_{1}$ and $q_{2}$.

11 Our proposal can be viewed as a refinement of the procedure of BIOCLIM to define

12 different levels of suitability, relaxing the independence assumption. Indeed, the way

13 BIOCLIM differentiates suitability levels in the bounding box of $P$, using percentiles of

14 each environmental variable individually, is actually the output of a depth function that

15 can be read as follows. The environmental suitability of a location corresponding to point

$16 q$ on the environmental space, is twice the value of $d_{q}^{\prime}(P)=m^{\prime} / k$, where $m^{\prime}$ is the

17 minimum number of points to remove from $P$ so that $q$ is not in the minimum

18 hyperrectangle containing the remaining points of $P$. The multiplication by two is a

19 rescaling so that the environmental suitability of points in the most inner hyperrectangle

20 equals one. Figure 2B depicts the regions of points with equal $d$ ' values corresponding to

21 the set of 10 points of $\mathbb{R}$ represented by “ ” (the same points as in Figure 2A). Note

22 that the regions of points for which $d^{\prime}$ 'is greater than or equal to $m / k$, ', are also

23 nested, i.e., $\mathbb{R}={ }^{\prime} \supset^{\prime} \supset \cdots \supset^{\prime}$, where ' $=\quad-$. The region ${ }^{\prime}(m \geq 1)$ is 
1 the intersection of all bounding boxes of the subsets of $P$ with $k-(m-1)$ points. Therefore,

2 we have: i) $M^{\prime}=M$ if $n=1$ and $M^{\prime}>M$ for $\geq 2$;ii) ' $\supset$, for $m=1, \ldots, M$, since the

3 bounding box of a set of points always contains the convex hull of the same set; or,

4 equivalently, for any $q, d^{\prime}{ }_{q}(P)=m^{\prime} / k \geq m / k=d_{q}(P)$.

(Figure 2)

6 Both procedures differentiate the outer polygon in nested regions of increasing suitability

7 for the interior, $\mathrm{CH}$-Tukey producing tighter regions than BIOCLIM. Points outside the

8 outer polygon are considered unsuitable; points in the margins have small suitability,

9 while the largest suitability is assumed to occur in the inner regions.

\section{Assessing the overlap of niches}

12 Several indices have been proposed to assess the niche overlap between two species, by

13 comparing the respective suitability (or presence/absence) on a finite number of points,

14 which correspond to cells of the geographical area (see Warren et al., 2008, for a survey).

15 However, to assess the overlap of the environmental niches of two species all points of

16 the environmental space should be considered and not only the projection into the cells of

17 a map. This is an issue that is particularly important in climate change scenarios. Villéger

18 et al. (2011) proposes to evaluate the functional dissimilarity between two (or more)

19 species assemblages, considering the volumes of the union and of the intersection of the

20 convex hulls of the points representing each species assemblage in the space of their

21 functional traits. This approach can be easily transposed to the evaluation of the overlap

22 between the environmental niches of two species, giving an overlap index defined as the 
1 ratio between the volume of the intersection and the volume of the union of the convex

2 hulls of the occurrences of both species. This measure can be interpreted as the Jaccard

3 index for the volume and is the natural way to estimate niche overlap when using the $\mathrm{CH}$ -

4 approach to describe the niche. It has the disadvantage of not distinguishing between

5 intersections occurring in regions of high suitability and those occurring in low suitability

6 areas of the niches.

7 As Tukey depth divides the niche into a finite number of iso-suitability regions, it allows

8 to generalize Pianka and Jaccard indices incorporating volumes and suitability.

9 Consider two species A and B, and the partition of the environmental space into and

10 regions of (non zero) iso-depth for each species. Denote by $0=$ !"\#\$ $<$ !"\#\$<

$11 \cdots<$ ! , \#\$ and $0=$ !" $(\$<!$ !" $\$<\cdots<$ ! , "(\$ all possible values of suitability for

12 species A and B, respectively, and let ${ }^{*}$, with $(i, j) \neq(0,0)$, be the volume of the (possibly

13 disconnected) region composed by all the environments having suitability !+"\#\$ for

14 species A and!",(\$ for species B, and * arbitrary. The volumes can be arranged in a

$15(+1)$ by $(+1)$ matrix ${ }_{-}=\cdot_{+}^{*} /$. This matrix contains all the information on

16 intersections of the environmental niches, taking into account distinct levels of suitability

17 for both species. Note that, if !+"\#\$ and !", \$ only take 0-1 values, i.e. resulting from

18 binary prediction models, such as the $\mathrm{CH}$-approach, . $=._{+}^{*} /$ / is a 2 by 2 matrix, where

$19 *$ is the volume of the intersection of the niches, and $*(*)$ is the volume of the

20 niche of species A (B) not shared by species B (A).

21 From $M_{V}$ we define the matrix $M_{\mathrm{A}}\left(M_{\mathrm{B}}\right)$ of the asymmetric overlap of species B (A) on A

22 (B), multiplying the row $i$ of $M_{V}$ by $!_{+} " \# \$$ (the column $j$ of $M_{V}$ by !"(\$). Each entry $(i, j)$ 
1 of matrix $M_{\mathrm{A}}\left(M_{\mathrm{B}}\right)$ is the volume ${ }_{+}$, of the region with iso-suitability !+"\#\$, for species $\mathrm{A}$

2 and !,"(\$ for species B, weighted by !+"\#\$ (!", $\$)$.

3 We propose to use the matrices above to define two overlap indices. The first is

4

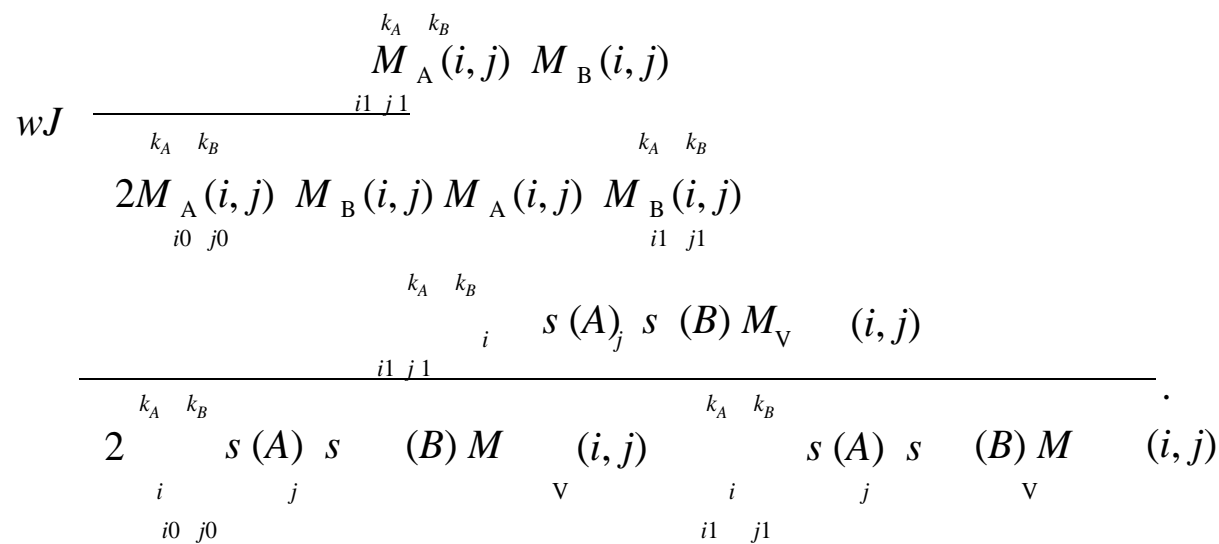

5 Index $w J$ incorporates the different levels of suitability on the ratio of the volumes of the

6 intersection and of the union of the niches. If !+"\#\$ and !,"(\$ only assumes values 0 or 1 ,

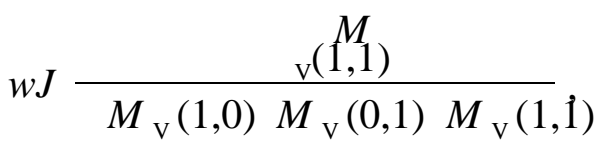

10 which is the Jaccard index for the volume. For the particular case when suitability is

11 given by $\mathrm{CH}$-approach, the niches are estimated by the convex hulls of the occurrences of

12 both species, and $w J$ becomes the index of Villéger et al. (2011).

13 When suitability is obtained with Tukey depth, $w J$ distinguishes between intersections

14 occurring in regions of high suitability and those occurring in low suitability areas of the 15 niches. 
1 Figure 3 illustrates iso-suitability regions for each of three hypothetical species A, B and

2 B' (top) in the same environmental space, and how they are arranged together (bottom).

3 The volumes of the intersections/unions of the convex hulls for A and B, and for A and

4 B' are exactly the same. Thus, Jaccard index returns the same value $(0.2179)$ for both

5 pairs. Index $w J$ gives 0.3579 for pair $(\mathrm{A}, \mathrm{B})$, where intersection occurs mainly in the

6 regions of high suitability, and 0.1604 for pair (A, B') where the regions of high

7 suitability almost do not overlap.

(Figure 3)

9 The second overlap index we propose is a cosine index, which is an adaptation of Pianka

10 index used to measure resource overlap between two species (Pianka, 1973). Consider

11 matrices $M_{\mathrm{A}}$ and $M_{\mathrm{B}}$ as vectors of length $(+1) \times(+1)$. The $\cos$ index is defined as the

12 cosine of the angle between these vectors.

13

14

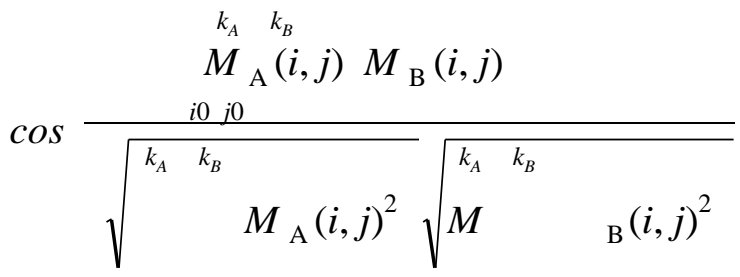

$\begin{array}{llll}i 0 & j 0 & i 0 & j 0\end{array}$

$s_{i}(A) s_{j}(B)\left(M_{\mathrm{V}}(i, j)\right)^{2}$

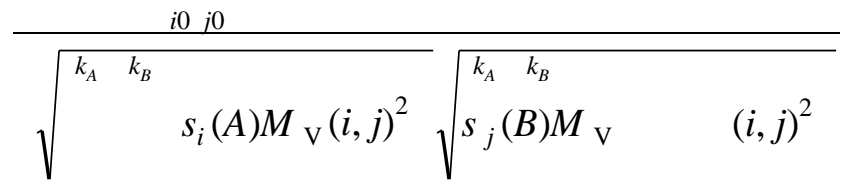

$\begin{array}{llll}i 0 & j 0 & i 0 & j 0\end{array}$

16 When the niches do not overlap, vectors are orthogonal and $\cos =0$. When high and low

17 suitability occur in the same regions for both species, $\cos \approx 1$. 


\section{ILLUSTRATIVE EXAMPLE}

\section{The climatic suitability of Quercus taxa in the Iberian Peninsula}

4 As an example of application of the above-described $\mathrm{CH}$-Tukey procedure, we computed

5 climatic suitability for Quercus taxa occurring in the Iberian Peninsula.

6 Quercus spp. are widespread in the Northern Hemisphere (Manos et al., 1999). In the

7 Iberian Peninsula Quercus taxa occur in most of the territory being an important

8 economic and conservation target (see Bugalho et al., 2011; Correia et al., 2017). For

9 example, several of the local economies are dependent on cork extraction, cork represents

$102.5 \%$ of Portugal exports, and acorns from Quercus rotundifolia and Q. suber are

11 particularly important in many animals' diet (Rodríguez-Estévez et al., 2009; Bugalho et

12 al., 2011). Q. robur is an important forestry species. Q. faginea and $Q$. pyrenaica are

13 valuable to local communities for the production of firewood (Gonçalves et al., 2012;

14 Cerqueira et al., 2013).

15 In this paper we focus our analyses on six species/subspecies: $Q$. rotundifolia $(=Q$. ilex

16 subsp. ballota), Q. suber, Q. pyrenaica, Q. faginea subsp. faginea, Q. faginea subsp.

17 broteroi and Q. robur. Occurrences were obtained from the Proyecto Anthos (Anthos,

18 2011) and from the Herbarium "João de Carvalho e Vasconcellos" (Lisbon, LISI) and

19 include a mix of point and grid-based data of several natures and types. Positional

20 accuracy of the species observations data was analysed and improved when appropriate.

21 We used four climatic variables known to affect and limit species distributions,

22 downloaded from the Worldclim website (Hijmans et al., 2005; 
$1 \mathrm{http} / / / \mathrm{www}$.worldclim.org) at the $1 \mathrm{~km} \times 1 \mathrm{~km}$ grid cell resolution. Two variables involve

2 temperature (isothermality and the minimum temperature of coldest month) and two

3 variables involve precipitation (annual precipitation and the precipitation of driest

4 quarter).

5 For each selected taxon, we applied the $\mathrm{CH}-\mathrm{Tukey}$ and the BIOCLIM procedures in order

6 to produce geographical maps distinguishing suitability levels. The Tukey depth of each

7 site within the Iberian Peninsula (ca. $1 \mathrm{~km} \times 1 \mathrm{~km}$ resolution), with respect to the

8 occurrences of each taxon, was determined using the function depth from the R package

9 depth version 2.0-0 (Genest et al., 2012). The depth values were then rescaled dividing

10 by $0 "-3 \$ / 23$, where $k$ is the number of occurrences of the taxon, thus obtaining a

11 normalized Tukey depth. The BIOCLIM suitability associated to each site was obtained

12 using the functions $b c$ and predict from the R package dismo version 1.1-1 (Hijmans et

13 al., 2016).

14 The maps clearly show that the inland non-white areas for CH-Tukey, (Figure 4) are

15 contained in the inland non-white areas for BIOCLIM, ' (Figure 5), denoting the fact

16 that the convex hull is a tighter envelope than the hyperrectangle.

17 A robust validation of these models imply controlled experiments using a small set of

18 factors considered limitative of species fitness. Given that these experiments are typically

19 time-consuming, expensive and therefore are seldom performed, expert knowledge is a

20 valuable source of information to discuss niche predictions.

21 The obtained spatial extents are quite consistent with experts' knowledge (see e.g. Rivas-

22 Martínez \& Sáenz Laín, 1991), specially for the CH-Tukey approach (e.g. Q. robur in the

23 NW of the Peninsula, Q. faginea subsp. broteroi in the SW, Q. suber mainly in the W, 
1 while the other three species occur mostly in the interior parts of the Peninsula). For the

2 BIOCLIM procedure such spatial coincidence is not so evident and can only be

3 approximated if the lower levels of suitability are discarded.

4 For some taxa (Q. suber, Q. rotundifolia and both $Q$. faginea subspecies) the maps show

5 a low density of occurrences in regions of predicted high suitability. This may result,

6 among other possible reasons, from: i) missing relevant environmental variables (e.g. soil

7 characteristics or other climatic variables) in the model; ii) incomplete sampling of

8 occurrences in the corresponding geographic areas; iii) some ecological factors that lead

9 to the exclusion of the species from that area (e.g. competition, parasitism, disease); or

10 even iv) local extinction due to human overuse of Quercus forests, conversion to

11 agricultural fields, managed forests, pastures or urban fabric. In the case of $Q$. faginea

12 subsp. faginea and Q. suber it is quite plausible that a soil-related environmental variable

13 is missing in the model, as the former is known to prefer base-rich or clay-rich soils,

14 while the latter is calcifugous (Franco, 1990). In the case of Q. faginea subsp. broteroi,

15 the biogeographic disjunction that the occurrence points show (i.e. one cloud of

16 occurrences concentrated in the west of Portugal and a second cloud in the central part of

17 Spain) may lead to an unoccupied niche core. The findings of Silva et al. (2014),

18 mentioned above, can be used to detect those less frequent distribution patterns within the

19 niche, previously to modelling.

20 In general, with the CH-Tukey approach, the maximum suitability obtained for each of

21 the studied Quercus taxa is relatively low. This is not problematic a priori and might give

22 further structural information on the species niche and on the study area, as: i) the

23 maximum suitability level of 1 , might not be achieved for some configurations of 
1 occurrence points in the environmental space; ii) when achieved, the volume of the

2 region with suitability 1 can be very small; iii) even when the volume of the Tukey

3 median region is conspicuous, it may correspond to combinations of the $n$ variables that

4 do not come into existence within the study area.

(Figure 4)

(Figure 5)

\section{Quantifying the environmental niche overlaps of Iberian Quercus taxa}

9 In the space defined by the four environmental variables (isothermality, minimum

10 temperature of coldest month, annual precipitation and precipitation of driest quarter), we

11 uniformly generated $N=45 \times 10^{6}$ points within the bounding box of the occurrences of all

12 taxa, and computed the Tukey suitability of each of these points with respect to the

13 occurrences of each taxon. To assess niche overlap for each pair of taxa, A and B, we

14 estimated the volumes ${ }_{+}^{*}$, for " $4,6 \$ \neq " 0,0 \$$, as the number of points with Tukey

15 suitability !+"\#, for taxon A, and Tukey suitability !",(\$, for taxon B, divided by $N$.

16 Results for the two proposed overlap indices $(w J$ and $\cos )$ are presented in Table 1.

(Table 1)

18 Both $w J$ and $\cos$ showed that $Q$. robur and Q. pyrenaica presented the highest overlap,

19 while $Q$. robur and $Q$. faginea subsp. broteroi presented the lower overlap.

20 In order to visualize graphically the relative positions of occurrences and iso-depth

21 regions of the pairs of taxa that, in the 4-dimensional space, were more separated $(Q$.

22 robur vs. $Q$. faginea subsp. broteroi), and less separated (Q. robur vs. $Q$. pyrenaica) 
1 (Table 1), we projected the occurrences of each of these taxa in the 2-dimensional

2 environmental space of variables minimum temperature of the coldest month and

3 precipitation of the driest quarter (Figure 6). Computing the overlap indices in this 2-

4 dimensional space, we obtained $w J=0.9177$ and $\cos =0.3450$ for the first pair $(Q$. robur,

$5 Q$. pyrenaica $)$ and $w J=0.3274$ and $\cos =0.0283$ for the second pair $(Q$. robur, $Q$. faginea

6 subsp. broteroi). These values agree with the graphs of Figure 6, where it is shown a clear

7 overlap of the niches of $Q$. robur and $Q$. pyrenaica while the niche of $Q$. faginea subsp.

8 broteroi is mainly concentrated in the peripheral areas of the $Q$. robur niche.

9 In general, Table 1 shows values that are coherent with the known regional co-occurrence

10 patterns of the studied Quercus (see e.g. Rivas-Martínez \& Sáenz Laín, 1991; Capelo et

11 al., 2007). For example, $Q$. robur co-occurs frequently with $Q$. pyrenaica in the highlands

12 of the northwest of the Iberian Peninsula, and with $Q$. suber in the lowlands. Q. pyrenaica

13 co-occurs with $Q$. rotundifolia in the continental plateaux of the Iberian Meseta and with

$14 Q$. suber in the warmer areas of the centre-south. Q. rotundifolia and $Q$. suber co-occur in

15 the SW of the Peninsula. The referred pairs correspond both to the highest values of the

16 indices shown in Table 1 and to intermingled vegetation series mapped as a mosaic in the

17 work of Capelo et al. (2007).

(Figure 6)

\section{Comparing niche geometries}

20 In order to illustrate and compare niche geometries produced by different approaches, we

21 compared outputs from CH-Tukey to BIOCLIM, and also MaxEnt (Phillips et al., 2006).

22 MaxEnt is one of the most used modelling algorithms, to obtain species environmental

23 suitability maps (Ahmed et al., 2015). We ran these three models using the two variables 
1 referred above (minimum temperature of the coldest month and precipitation of the driest

2 quarter) and depicted their outputs both in the environmental and in the geographic space

3 (Figure 7) for Q. robur, Q. pyrenaica and Q. faginea subsp. broteroi. Default MaxEnt

4 parameterization was used.

5 Inspecting Figure 7 (B, C, D) we can visualize how BIOCLIM relies on the assumption

6 that ecological variables are independent in their action on the species, producing

7 rectilinear niche geometries, while $\mathrm{CH}-\mathrm{Tukey}$ relaxes it, adjusting its outer boundary and

8 the inner suitability levels to the known occurrences. Both BIOCLIM and CH-Tukey are

9 built upon the "range" notion, implicit in Hutchinson's seminal work, i.e., if you select

10 two points from the species niche representing environmental conditions known to be

11 suitable for that species, you should not expect a decrease in suitability between these two

12 points, at least while only those variables are considered. MaxEnt neglects this, producing

13 not only unbounded geometries, but also allowing (visible for the three tested taxa) a

14 decrease in suitability between two points of the niche. Although relying on unimodal

15 geometries, BIOCLIM and CH-Tukey approximate better Hutchinson's notion of optimal

16 versus suboptimal niche regions than the geometry produced by MaxEnt, which proposes

17 high suitability values for unbounded or extrapolative areas (see "clamping" in Merow et

18 al., 2013; check Q. faginea subsp. broteroi output in Figure 7D, values of min

19 Temperature greater than $10.0^{\circ} \mathrm{C}$ or smaller than $-10.0^{\circ} \mathrm{C}$ and Precipitation around 50

$20 \mathrm{~mm}$ ). This is in agreement with the known limitations of correlative models concerning

21 extrapolation (Merow et al., 2013). According to the same authors, MaxEnt can produce

22 intricate model outputs of hard ecological interpretation, especially when regularization is

23 used. This is apparent in the sudden shifts in suitability observed in the MaxEnt output 
1 (within the species niche). Even if some shifts could relate to interesting or interpretable

2 facts (e.g. coming from the background data), we believe that they deviate this modelling

3 technique from the seminal ecological theory.

\section{(Figure 7)}

6 A thorough and careful setting of the input parameters in MaxEnt can improve the

7 ecological interpretation of the output (Merow et al., 2013), but such parameterization is

8 not always achievable using presence-only data coming from usual databases. Even so,

9 MaxEnt produces geographical maps that are closely linked to the known occurrences of

10 points (see Figure 7A for the occurrence points of each taxa), which is expected from a

11 correlative approach. This tends to hinder fundamental discussions, such as: i) niche

12 boundaries and niche optimality; ii) interpolation vs. extrapolation of the observations;

13 and iii) possible discrepancies between niche optimality and the geographic distribution

14 (e.g. niche is highly suitable according to the studied environmental variables, but there

15 are no occurrences in the corresponding geographic regions, possibly indicating that other

16 important variables lack in the model).

17 Incorporating putative niche voids directly in the modelling approaches has been

18 considered useful, especially while addressing niches using real-world examples (Walker

19 \& Cocks, 1991; Blonder et al., 2014). Nevertheless, even when dealing with real-world

20 examples, general niche premises and properties should hold (e.g. the "range" notion,

21 niche boundaries and optimality) and these can be consistently addressed and explored

22 using appropriate methodological approaches such as BIOCLIM and CH-Tukey. 


\section{CONCLUDING REMARKS}

3 The $\mathrm{CH}$-Tukey procedure allows the construction of environmental suitability maps, as

4 shown for the selected Quercus taxa, differentiating iso-suitability levels within the CH-

5 approach. Such levels are drawn from occurrence data only, in the selected environmental

6 hyperspace, using normalized Tukey depth, and can, subsequently, be used to derive

7 overlap measures coherently.

8 Carpenter et al. (1993) listed four disadvantages linked with the $\mathrm{CH}$-approach, two

9 related to computational difficulties which are no longer a problem for current computers.

10 The other two were related to the tightly constrained envelope produced by the $\mathrm{CH}$ -

11 approach and the exclusion of many similar sites (close to, but outside, the convex hull

12 boundary). But this may be arguable since the convex hull is a natural boundary between

13 interpolation and extrapolation (see Hirzel \& Le Lay, 2008). We stress that the CH-Tukey

14 procedure is appropriate to interpolate from the input data, as the model simply considers

15 unsuitable all environmental combinations outside the convex hull (i.e. it does not

16 extrapolate). Nevertheless, similar sites in the immediate outside neighbourhood of the

17 convex hull, which are considered unsuitable in the $\mathrm{CH}$-Tukey procedure, could be

18 modelled separately with other techniques, in a coupled approach, assuming that

19 extrapolation is acceptable.

20 As shown in the maps obtained with $\mathrm{CH}-$ Tukey methodology, it is possible that higher

21 suitability regions have low density of occurrences (see e.g. $Q$. faginea subspecies). This

22 results from the combinatorial-geometrical nature of the approach that does not rely so

23 strongly on point density to predict suitability. As the procedure is geometrically 
1 interpretable, hypotheses can be sketched from the obtained patterns, given the used

2 environmental variables, and tested subsequently. This interpretability ensures a seamless

3 relationship with Hutchinsonian niche concept, a feature frequently asked by ecologists

4 and epistemologists (Austin, 2002; Elith \& Leathwick, 2009; Brewer et al., 2016).

5 In conclusion, the $\mathrm{CH}-\mathrm{Tukey}$ procedure presents several relevant features that can be

6 considered advantages in some ecological niche modelling contexts, such as:

7 i) The modelling process is easily interpretable geometrically, with precise definition of

8 the estimated niche boundaries.

9 ii) It uses only presence data and does not depend on the background data.

10 iii) The suitability levels are invariable with respect to the ecological variables units.

11 iv) The regions of the multivariate space corresponding to high Tukey depth values are

12 robust to outliers, in accordance to what occurs with the univariate median of a set of

13 values.

14 v) It produces ordinal predictions, decomposing the niche into a finite number of iso-

15 suitability levels, permitting the computation of consistent overlap indices.

16 vi) A tight envelope is adjusted to the points cloud and, consequently, the independence

17 of the ecological variables action on the species is not assumed (i.e., allowing for

18 interactions between variables); moreover, the output of the procedure results uniquely

19 from interpolations of the input data.

20 The main disadvantage of the $\mathrm{CH}-$ Tukey procedure is that depth computation is time

21 demanding, and heuristic approximations may be required depending on the number of 
1 occurrence points used as input data and on the spatial resolution of the predictions.

2 Heuristics are already implemented in package depth (Genest et al., 2012).

\section{ACKNOWLEDGMENTS}

5 This work was supported by the Portuguese Foundation for Science and Technology

6 (FCT) through the projects UID/MAT/00297/2013, CMA (Centro de Matemática

7 Aplicada); UID/AGR/00239/2013, CEF (Centro de Estudos Florestais) under

8 FEDER/POCI; and PTDC/AAC-AMB/ 113394/2009. T. Monteiro-Henriques was

9 partially funded by a post-doctoral grant within Project SUSTAINSYS: Environmental

10 Sustainable Agro-Forestry Systems - NORTE-07-0124-FEDER-0000044 (ON.2-QREN-

11 FEDER and PIDDAC-FCT-MEC). D. Alagador was funded through the project

12 PTDC/AAG-GLO/3979/2014 via FEDER through the COMPETE program and national

13 funding. D. Alagador and F. C. Aguiar are also supported with FCT post-doctoral

14 fellowships (SFRH/BPD/104077/2014 and SFRH/BPD/112417/2015, respectively).

\section{AUTHORS' CONTRIBUTIONS}

17 JOC, TMH, MJM and PCS conceived the ideas and designed methodology; MJM, TMH

18 and MLC dealt with computational issues; TMH, PA, MJM and FCA collected, analysed

19 and interpreted the data; JOC, TMH, MJM and PCS led the writing of the manuscript.

20 DA, AF and MC revised it and contributed to the development of ideas. All authors

21 contributed critically to the manuscript. 
2 Ahmed, S.E., McInerny, G., O'Hara, K., Harper, R., Salido, L., Emmott, S. \& Joppa, L.N. (2015) Scientists and software - surveying the species distribution modelling community. Diversity and Distributions, 21, 258-267.

5 Anthos (2011) Sistema de información de las plantas de España. Real Jardín Botánico,

6 CSIC- Fundación Biodiversidad. Downloaded in November 16, 2011.

7 Austin, M.P. (2002) Spatial prediction of species distribution: an interface between ecological theory and statistical modelling. Ecological Modelling, 157, 101-118.

9 Blonder, B. (2017) Hypervolume concepts in niche- and trait-based ecology. Ecography, $10 \quad \mathbf{4 0}, 001-013$.

11 Blonder, B., Lamanna, C., Violle, C. \& Enquist, B.J. (2014) The n-dimensional hypervolume. Global Ecology and Biogeography, 23, 595-609.

13 Bond, W.J. \& Midgley, J.J. (2001) Ecology of sprouting in woody plants: the persistence niche. Trends in Ecology \& Evolution, 16, 45-51.

15 Booth, T.H., Nix, H.A, Busby, J.R. \& Hutchinson, M.F. (2014) BIOCLIM: the first species distribution modelling package, its early applications and relevance to most current MAXENT studies. Diversity and Distributions, 20, 1-9.

18 Brewer, M.J., O’Hara, R.B., Anderson, B.J., \& Ohlemüller, R. Plateau: A New Method for Ecologically Plausible Climate Envelopes for Species Distribution Modelling. Methods in Ecology and Evolution, 7, 1489-1502. 
1 Bugalho, M.N., Caldeira, M.C., Pereira, J.S., Aronson, J. \& Pausas, J.G. (2011) Mediterranean cork oak savannas require human use to sustain biodiversity and ecosystem services. Frontiers in Ecology and the Environment, 9, 278-286.

Busby, J.R. (1991) BIOCLIM - A Bioclimate Analysis and Prediction System. Nature Conservation: Cost Effective Biological Surveys and Data Analysis (ed. by C.R. Margules and M.P. Austin), pp. 64-68. CSIRO.

Capelo, J., Mesquita, S., Costa, J.C., Ribeiro, S., Arsénio, P., Neto, C., MonteiroHenriques, T., Aguiar, C., Honrado, J., Espírito-Santo, D. \& Lousã, M.F. (2007)

9
A methodological approach to potential vegetation modeling using GIS techniques and phytosociological expert-knowledge: application to mainland

15 Cerdeira, J.O., Monteiro-Henriques, T., Martins, M.J., Silva, P.C., Alagador, D. \&

18 Cerqueira, M., Gomes, L., Tarelho, L. \& Pio, C. (2013) Formaldehyde and acetaldehyde Franco, A.M.A. (2014) Mathematical contributions to link biota with environment. Journal of Vegetation Science, 25, 1148-1153.

Colwell, R. \& Futuyma, D. (1971) Measurement of Niche Breadth and Overlap. Ecology, 52, 567-576. 
1 Correia, R.A., Bugalho M.N., Franco A.M.A. \& Palmeirim J.M. Contribution of Spatially Explicit Models to Climate Change Adaptation and Mitigation Plans for a Priority Forest Habitat. Mitigation and Adaptation Strategies for Global Change, in press $1-16$.

5 Elith, J., Graham, C.H., Anderson, R.P., Dudík, M., Ferrier, S., Guisan, A., Hijmans, R.J.,

Franklin, J. (2010) Mapping Species Distributions: Spatial Inference and Prediction, Huettmann, F., Leathwick, J.R., Lehmann, A., Jin Li, Lohmann, L.G., Loiselle, B.A., Manion, G., Moritz, C., Nakamura, M., Nakazawa, Y., McC. Overton, J., Peterson, A.T. \& Phillips, S.J. (2006) Novel methods improve prediction of species' distributions from occurrence data. Ecography, 29, 129-151.

Elith, J. \& Leathwick, J.R. (2009) Species Distribution Models: Ecological Explanation and Prediction Across Space and Time. Annual Review of Ecology, Evolution, and Systematics, 40, 677-697.

Franco, J. do A. (1990) 3. Quercus L. *. Flora iberica: Plantas vasculares de la Península Ibérica e Islas Baleares (ed. by S. Castroviejo, M. Laínz, G. López González, P. Montserrat, F. Muñoz Garmendia, J. Paiva, and L. Villar), pp. 15-36. Real Jardín Botánico - Consejo Superior de Investigaciones Científicas, Madrid.

Franklin, J. (1995) Predictive vegetation mapping: geographic modelling of biospatial patterns in relation to environmental gradients. Progress in Physical Geography, 19, 474-499. Cambridge University Press, Cambridge. 
1 Genest, M., Masse, J.-C. \& Plante, J.-F. (2012) depth: Depth functions tools for multivariate analysis (version 2.0-0), http://CRAN.R-project.org/package=depth.

3 Gonçalves, C., Alves, C. \& Pio, C. (2012) Inventory of fine particulate organic compound emissions from residential wood combustion in Portugal. Atmospheric Environment, 50, 297-306.

6 Grubb, P.J. (1977) The maintenance of species-richness in plant communities: the importance of the regeneration niche. Biological Reviews, 52, 107-145.

8 Guisan, A. \& Zimmermann, N.E. (2000) Predictive habitat distribution models in 9 ecology. Ecological Modelling, 135, 147-186.

10 Hijmans, R.J., Cameron, S.E., Parra, J.L., Jones, P.G. \& Jarvis, A. (2005) Very High 11 Resolution Interpolated Climate Surfaces for Global Land Areas. International 12 Journal of Climatology, 25, 1965-1978.

13 Hijmans, R.J., Phillips, S., Leathwick, J. \& Elith, J. (2016) dismo: Species distribution modeling, http://CRAN.R-project.org/package=dismo.

15 Hirzel, A.H. \& Le Lay, G. (2008) Habitat suitability modelling and niche theory. Journal of Applied Ecology, 45, 1372-1381.

17 Hurlbert, S. (1978) Measurement of Niche Overlap and Some Relatives. Ecology, 59, 6777.

19 Hutchinson, G.E. (1957) Concluding remarks. Cold Spring Harbor Symposia on Quantitative Biology, 22, 415-427. 
1 Manos, P.S., Doyle, J.J. \& Nixon, K.C. (1999) Phylogeny, biogeography, and processes of molecular differentiation in Quercus subgenus Quercus (Fagaceae). Molecular Phylogenetics and Evolution, 12, 333-349.

4 Merow, C., Smith, M.J., Silander Jr, J.A. 2013 A practical guide to MaxEnt for modeling species' distributions: what it does, and why inputs and settings matter. Ecography, 36, 1058-1069.

7 Nix, H.A. (1986) A biogeographic analysis of Australian elapid snakes. Atlas of elapid snakes of Australia (ed. by R. Longmore), pp. 4-15. Australian Government Publishing Service, Canberra.

10 Pearman, P.B., Guisan, A., Broennimann, O. \& Randin, C.F. (2008) Niche dynamics in 11 space and time. Trends in Ecology \& Evolution, 23, 149-158.

12 Peterson, A.T., Soberon, J. \& Sanchez-Cordero, V. (1999) Conservatism of ecological 13 niches in evolutionary time. Science, 285, 1265-1267.

14 Phillips, S.J., Anderson, R.P., Schapire, R.E. (2006) Maximum entropy modeling of species geographic distributions. Ecological Modelling, 190, 231-259.

16 Pianka, E.R. (1973) The Structure of Lizard Communities. Annual Review of Ecology and Systematics, 4, 53-74.

18 Raxworthy, C.J., Ingram, C.M., Rabibisoa, N. \& Pearson, R.G. (2007) Applications of 19 Ecological Niche Modeling for Species Delimitation: A Review and Empirical 20 Evaluation Using Day Geckos (Phelsuma) from Madagascar. Systematic Biology, $21 \quad \mathbf{5 6}, 907$. 
1 Rivas-Martínez, S. \& Sáenz Laín, C. (1991) Enumeración de los Quercus de la Península Ibérica. Rivasgodaya, 6, 101-110.

3 Rocap, G., Larimer, F.W., Lamerdin, J., Malfatti, S., Chain, P., Ahlgren, N.A., Arellano,

9 Rodríguez-Estévez, V., García, A. \& Gómez, A.G. (2009) Characteristics of the acorns

12 Root, R.B. (1967) The Niche Exploitation Pattern of Blue-Gray Gnatcatcher. Ecological Monographs, 37, 317-350.

14 Roughgarden, J. (1972) Evolution of Niche Width. The American Naturalist, 106, 683718.

16 Schoener, T. (1974) Resource Partitioning in Ecological Communities. Science, 185, $27-$ 39.

18 Silva, P.C., Cerdeira, J.O., Martins, M.J. \& Monteiro-Henriques, T. (2014) Data depth for the uniform distribution. Environmental and Ecological Statistics, 21, 27-39.

20 Silvertown, J. (2004) Plant coexistence and the niche. Trends in Ecology \& Evolution, 19, $605-611$. 
1 Tsoar, A., Allouche, O., Steinitz, O., Rotem, D. \& Kadmon, R. (2007) A comparative evaluation of presence-only methods for modelling species distribution. Diversity \& Distributions, 13, 397-405.

4 Tukey, J.W. (1975) Mathematics and the picturing of data. Proceedings of the International Congress of Mathematicians, 2, 523-531.

6 Villéger, S., Novack-Gottshall, P.M. \& Mouillot, D. (2011) The multidimensionality of the niche reveals functional diversity changes in benthic marine biotas across geological time. Ecology Letters, 14, 561-568.

9 Walker, P.A. \& Cocks, K.D. (1991) HABITAT: A Procedure for Modelling a Disjoint Environmental Envelope for a Plant or Animal Species. Global Ecology and Biogeography Letters, 1, 108-118.

12 Warren, D.L., Glor, R.E. \& Turelli, M. (2008) Environmental Niche Equivalency Versus 13 Conservatism: Quantitative Approaches to Niche Evolution. Evolution, 62, 28682883.

15 Werner, E. \& Gilliam, J. (1984) The Ontogenetic Niche and Species Interactions in Size 16 Structured Populations. Annual Review of Ecology and Systematics, 15, 393-425.

17 Whittaker, R.H. \& Levin, S.A. eds. (1975) Niche: Theory and Application, Dowen, 18 Hutchinson \& Ross, Pennsylvania.

19 Zuo, Y. \& Serfling, R. (2000) General notions of statistical depth function. The Annals of $20 \quad$ Statistics, 28, 461-482. 


\section{BIOSKETCH}

2

3 The authors include mathematicians, ecologists and biologists from several Universities

4 and Research Centers, involved in a three-year multidisciplinary project (S-PerforMA:

5 Species Performance Modelling Algorithm - http://www.isa.utl.pt/proj/S-PerforMa/)

6 aiming at exploring issues related with the concept of niche using combinatorial and

7 geometric tools. 


\section{TABLES}

2

3 Table 1. Overlap ( $w J$ and cos indices) of all pairs of Quercus taxa in the four dimensional

4 space defined by the used environmental variables: isothermality, minimum temperature

5 of coldest month, annual precipitation and the precipitation of driest quarter.

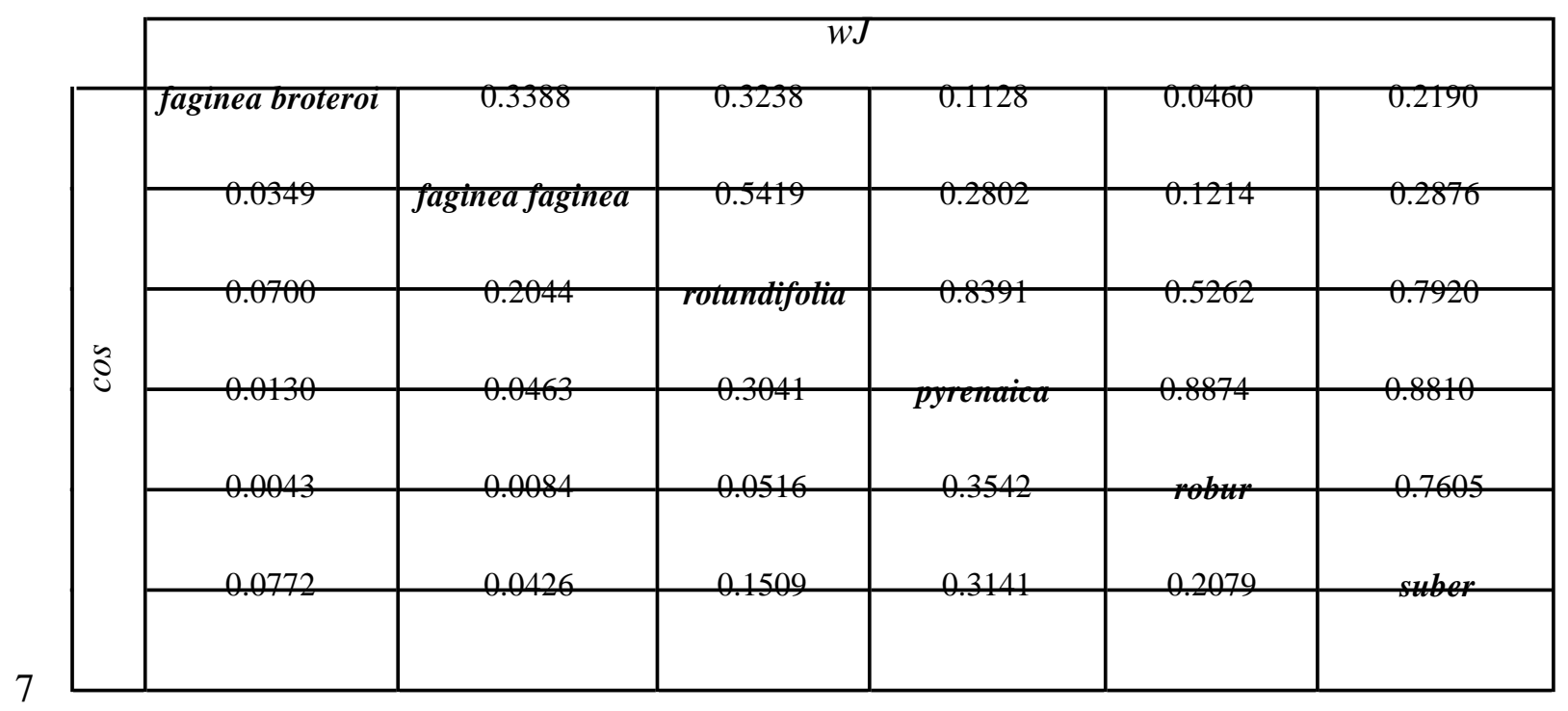




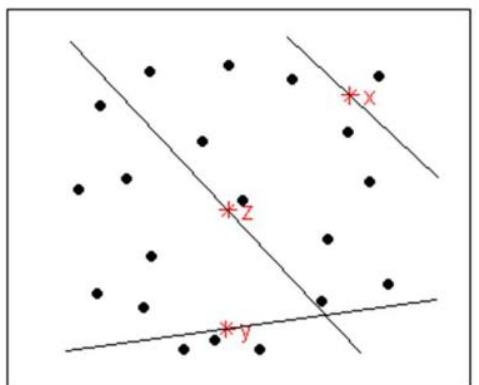

Figure 1. Illustration of the Tukey interiority of points $\mathrm{x}, \mathrm{y}$ and $\mathrm{z}$ (represented by $*$ ), with respect to the set $P$ of

20 points (represented by ), as the number of points of $P$ in the side of the line passing through $*$ which has the minimum number of points. Interiorities of $x, y$ and $z$ are 1,3 and 9 , respectively; $d_{x}(P)=1 / 20, d_{y}(P)=$ $3 / 20$, and $d_{z}(P)=9 / 20$. 


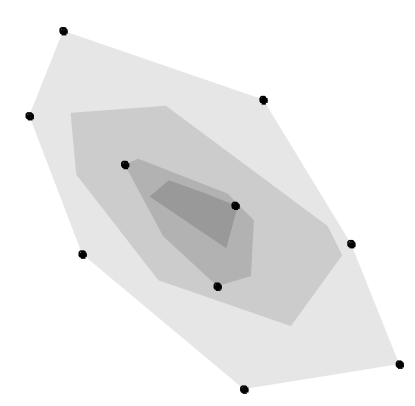

(A)

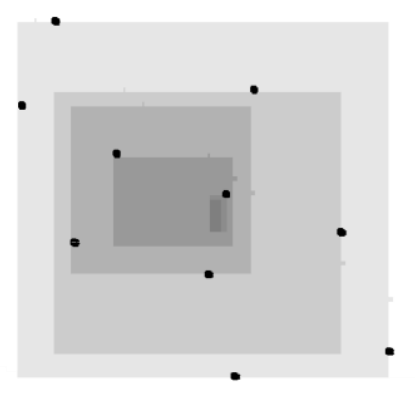

(B)

Figure 2. The 10 points represent occurrences of a species in a 2-dimensional environmental space. Shades of grey define areas of "iso-suitability". The suitability levels increase from the white $(=0)$ to the dark grey areas. The left panel (A) refers to CH-Tukey and the right panel (B) refers to BIOCLIM. 

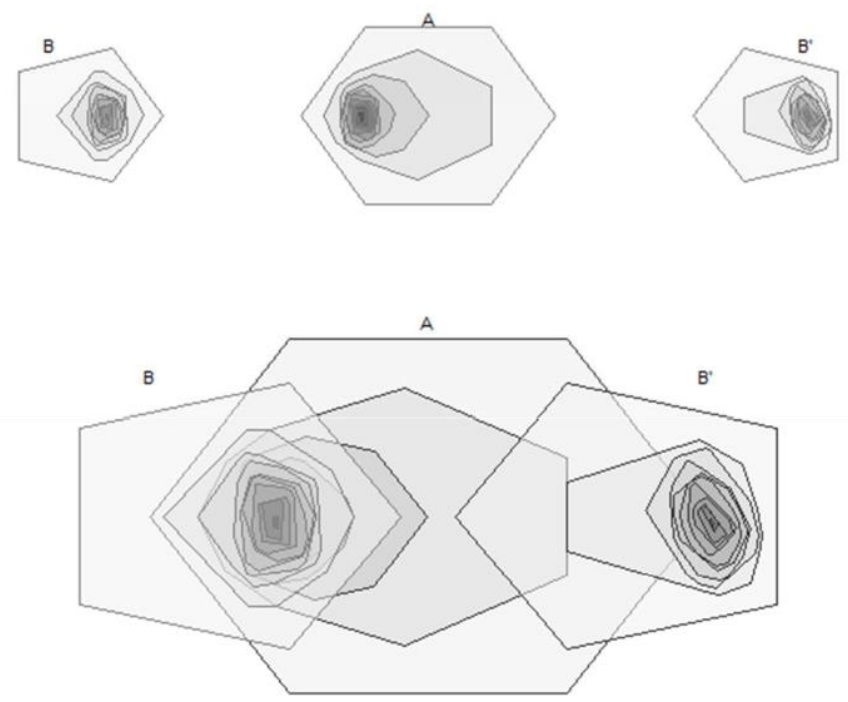

Figure 3. Niches and iso-depth regions of three species (A, B and B') in the same environmental space. Top row:

niches depicted isolated. Bottom row: overlapped niches. 

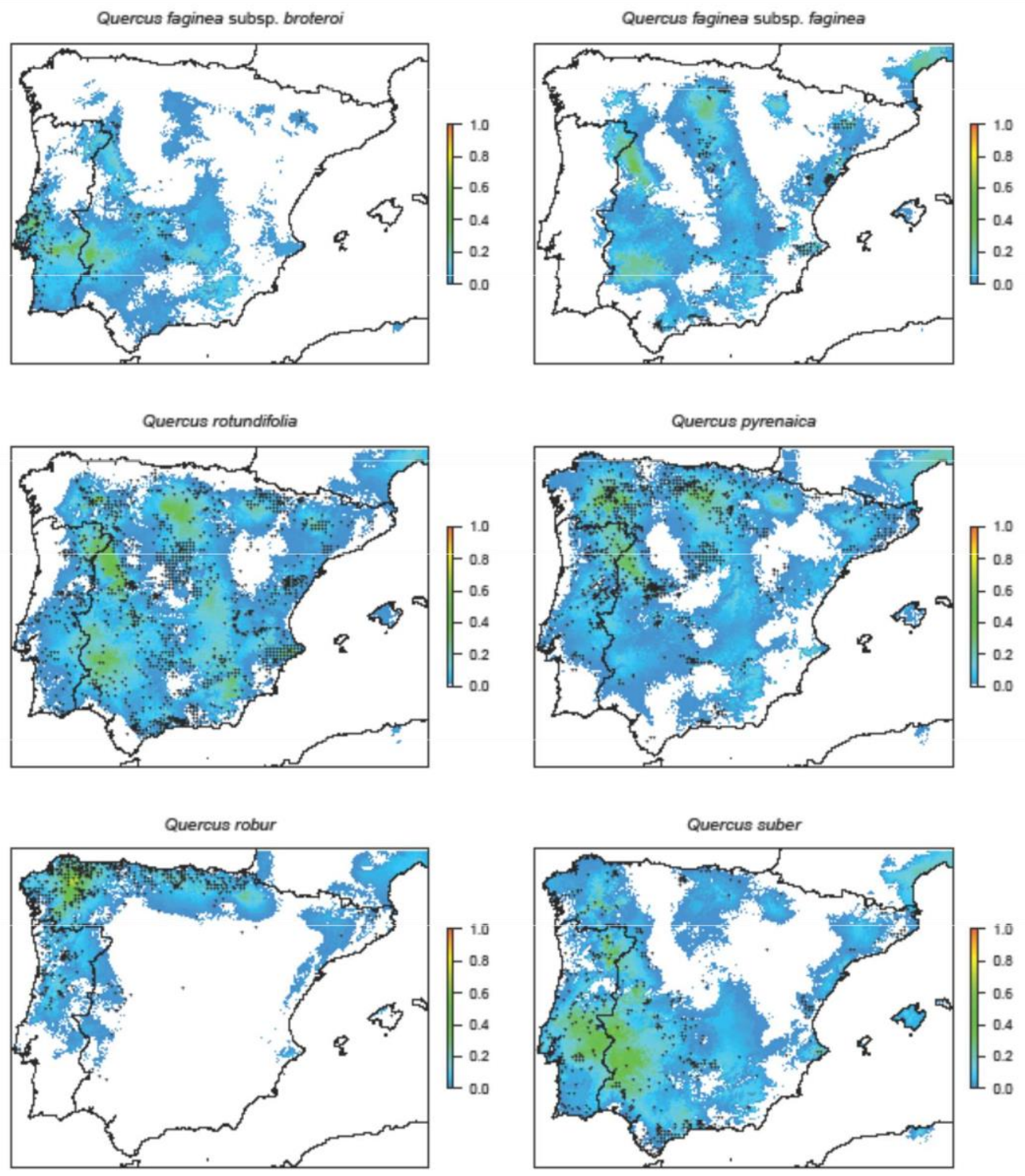

Figure 4. Environmental suitability maps for six Quercus taxa in the Iberian Peninsula obtained using the

$\mathrm{CH}-$ Tukey procedure, with four climatic variables. Dots represent occurrences. The white inland areas refer to points outside the convex hull of the occurrences of the corresponding taxon, in the considered 4dimensional environmental space. 

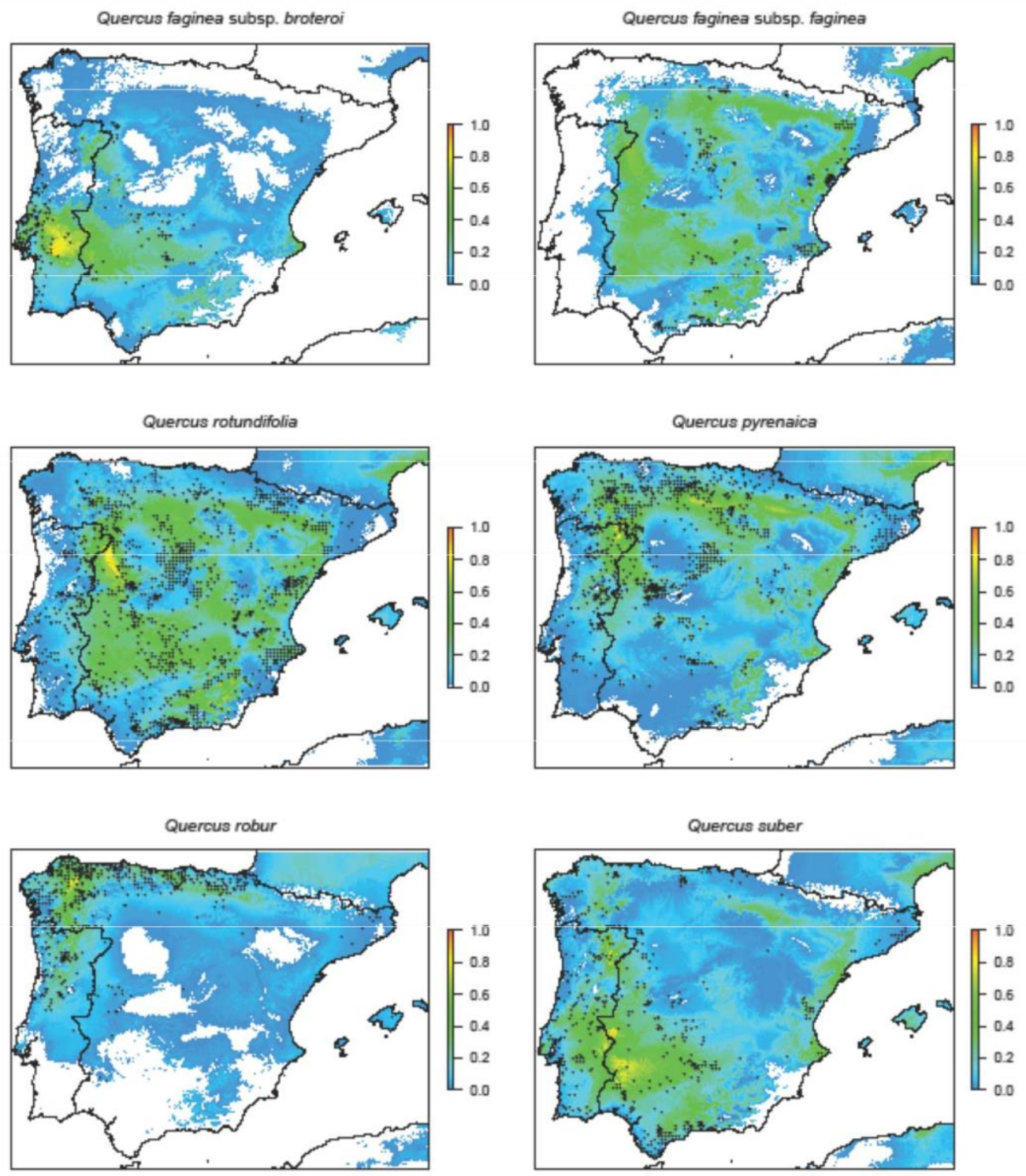

Figure 5. Environmental suitability maps for six Quercus taxa in the Iberian Peninsula obtained using the BIOCLIM model, with four climatic variables. Dots represent occurrences. The white inland areas refer to points outside the bounding box of the occurrences of the corresponding taxon, in the considered 4dimensional environmental space. 


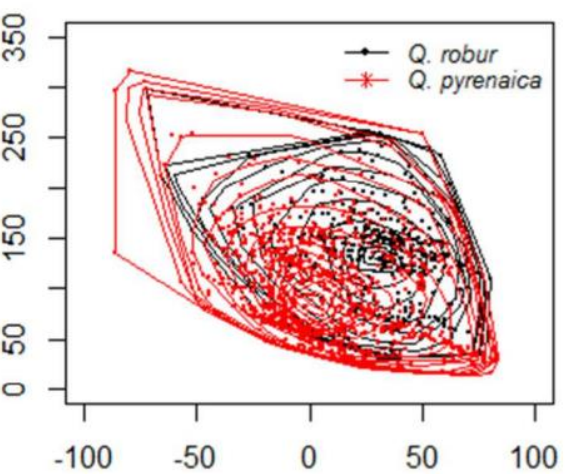

Min Temperature of Coldest Month $\left({ }^{\circ} \mathrm{C}^{*} 10\right)$

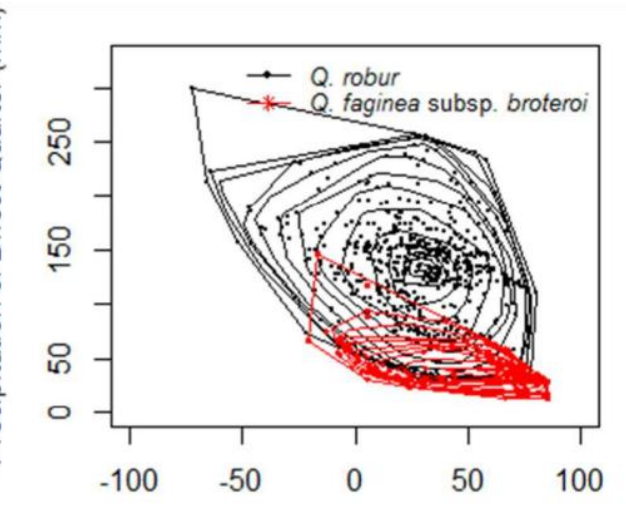

Min Temperature of Coldest Month $\left({ }^{\circ} \mathrm{C}^{*} 10\right)$

Figure 6. Occurrences and iso-depth regions of $Q$. robur and $Q$. pyrenaica in the left panel and of $Q$. robur and $Q$. faginea subsp. broteroi in the right panel, in the environmental space defined by the minimum temperature of the coldest month and precipitation of the driest quarter. Dots and stars correspond to occurrences, and the lines delimit iso-depth regions (regions with the same Tukey depth).

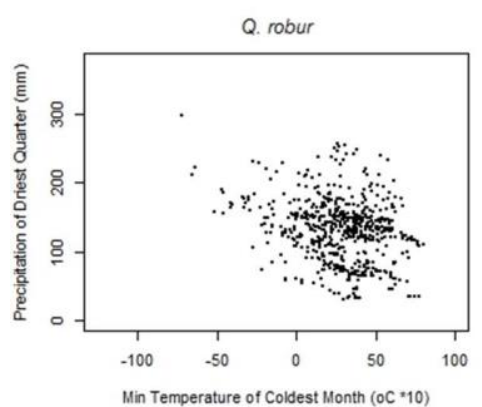

Q rosur

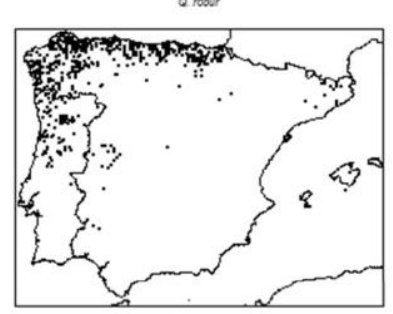

Q. pyrenaica

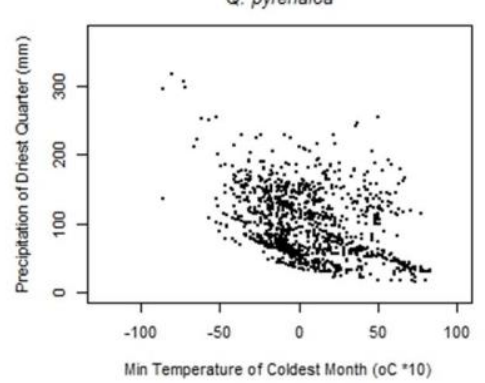

Q presaca

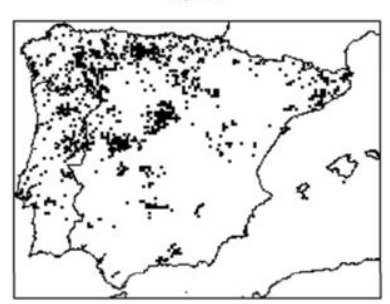

Q. faginea subsp. broteroi

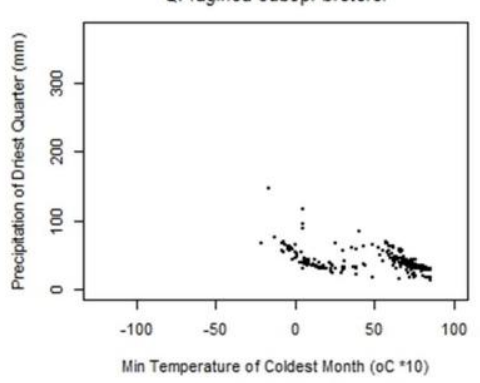

Q aginea suosp. orower

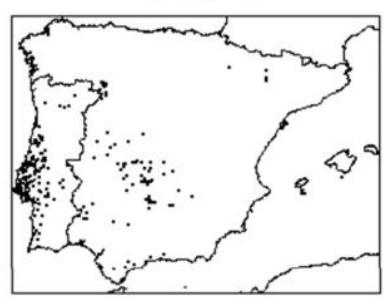

Figure 7A. Occurrences of $Q$. robur, $Q$. pyrenaica and $Q$. faginea subsp. broteroi in the environmental and the geographical spaces. 

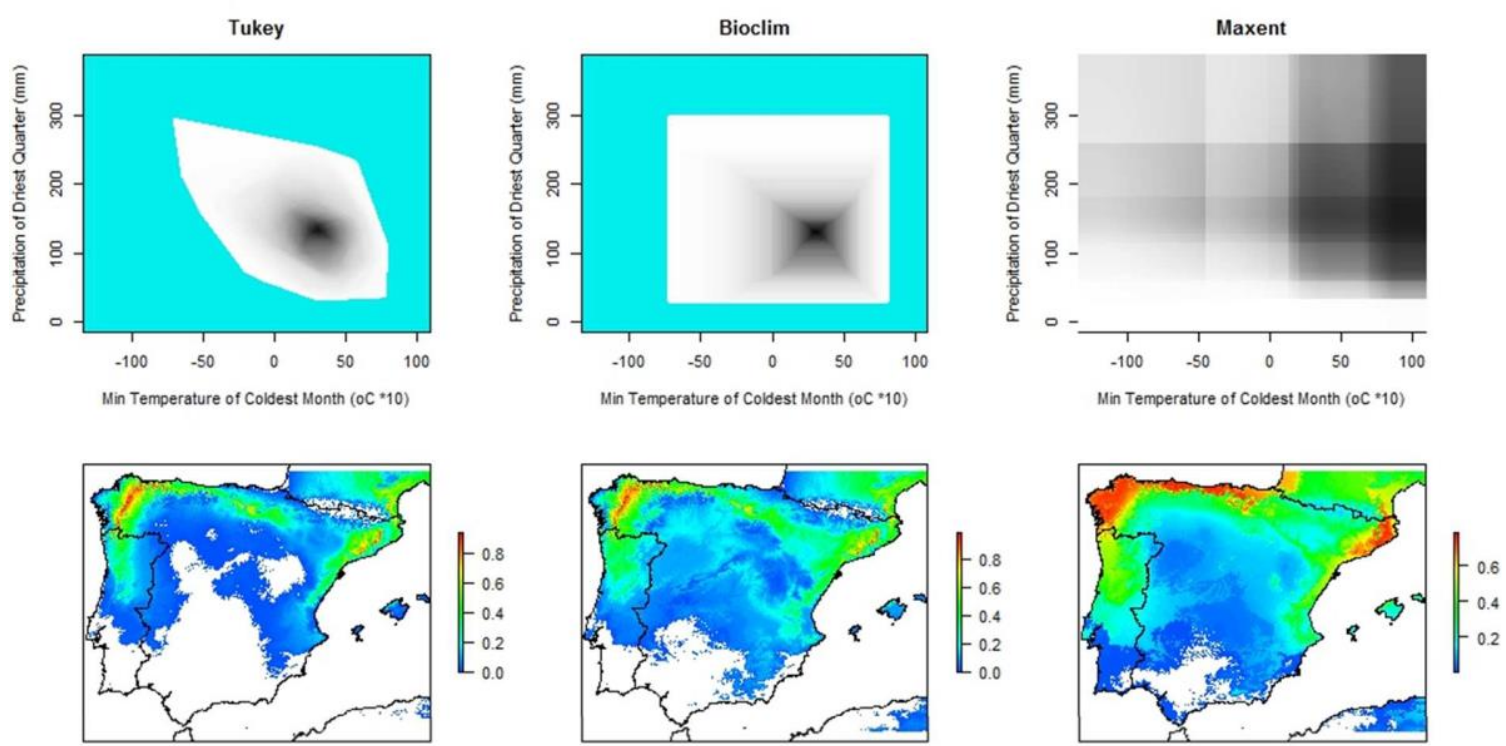

Figure 7B. Niches and suitability maps produced by CH-Tukey, BIOCLIM and MaxEnt for Q. robur.
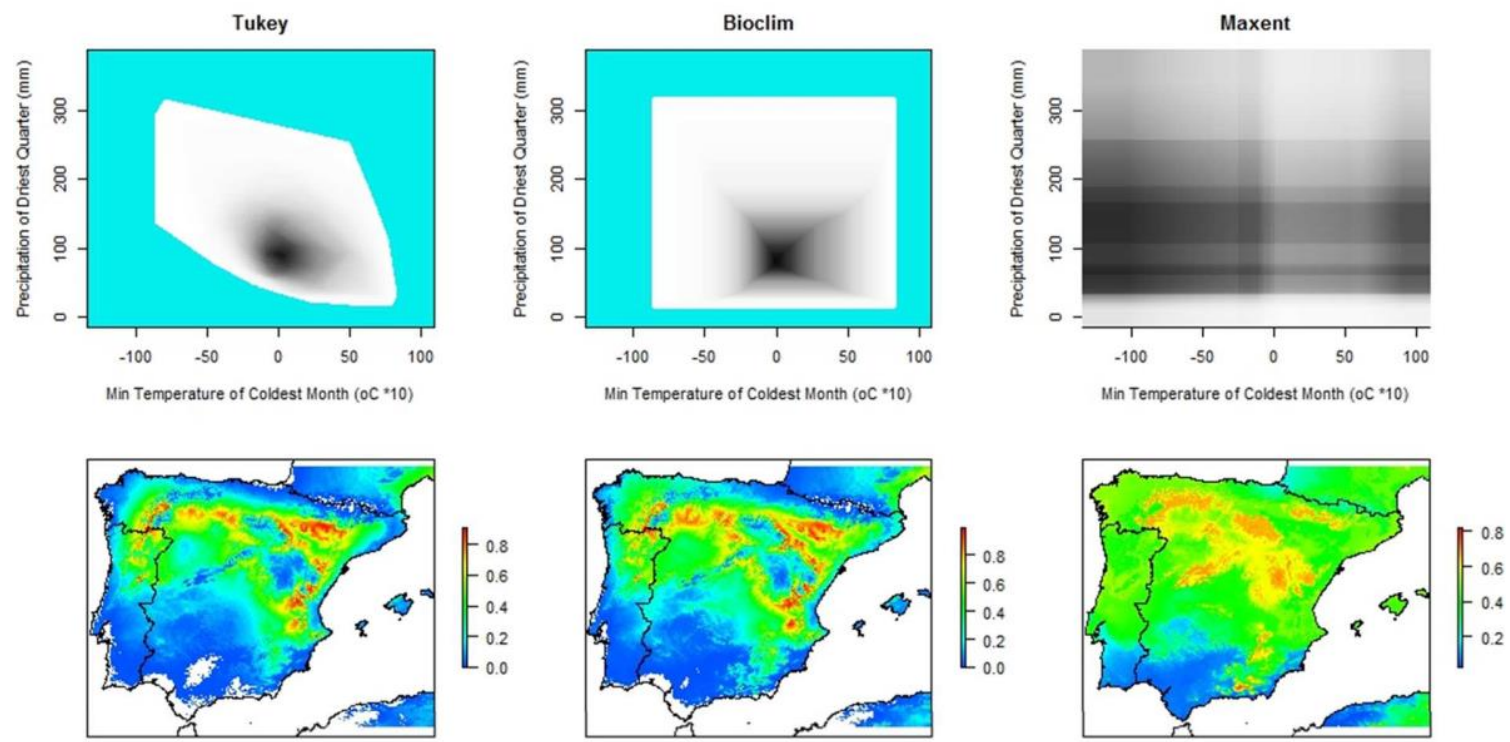

Figure 7C. Niches and suitability maps produced by CH-Tukey, BIOCLIM and MaxEnt for Q. pyrenaica. 

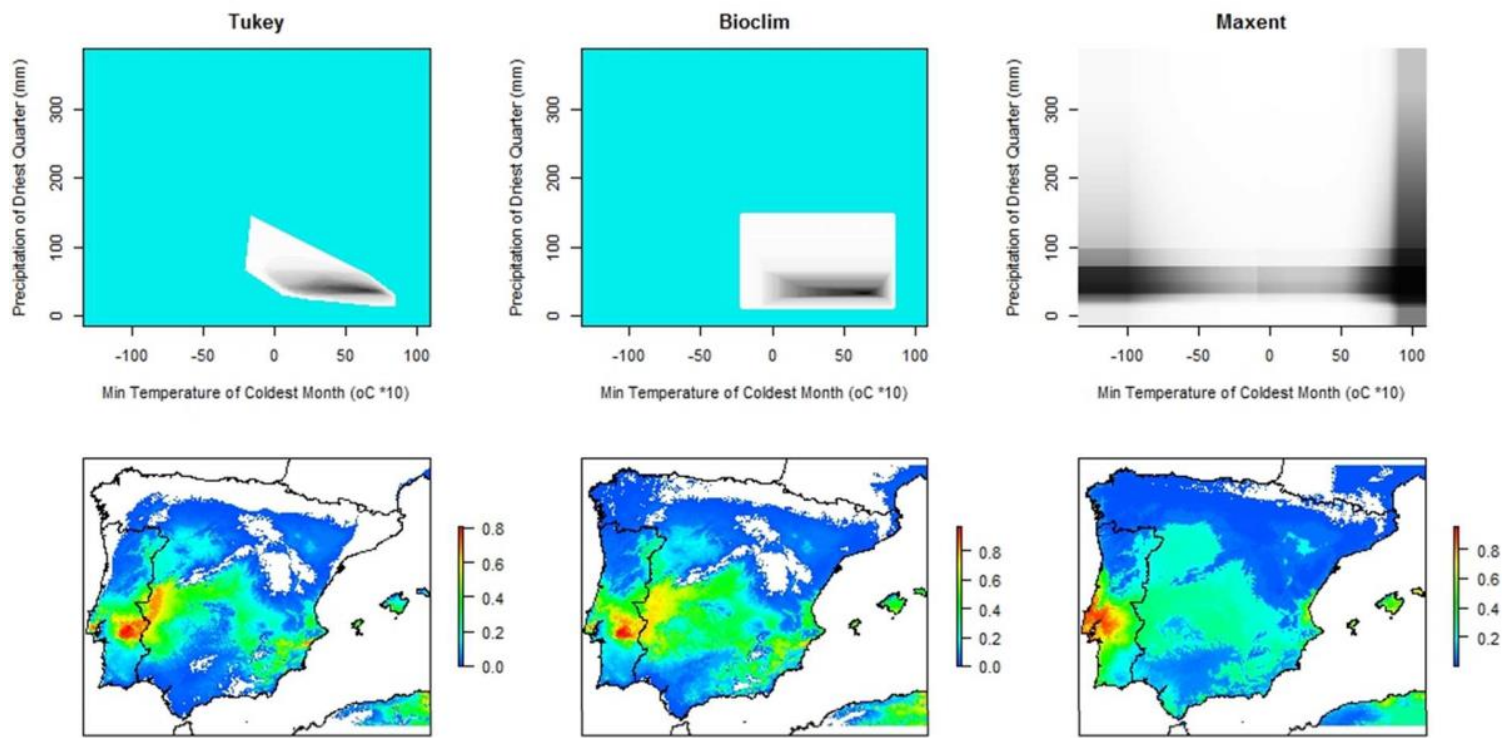

Figure 7D. Niches and suitability maps produced by CH-Tukey, BIOCLIM and MaxEnt for $Q$. faginea subsp. broteroi. 